\title{
CORRESPONDENCE
}

Turnround times in a microbiology laboratory

Drs Rogers, Bywater, and Reeves used a "simple method" to determine turnround time in a microbiology laboratory.' Their 11 week survey yielded data on only 140 samples. The authors rightly look forward to the computerisation of pathology services. As our own laboratory computer records when the specimen is received and results reported, it is a truly simple matter to analyse the laboratory turnround time of all specimens, or indeed any subgroup. As an example, we assessed the turnround time for urine specimens from the first week in March. Specimens were logged in between $09.00 \mathrm{~h}$ and $17.30 \mathrm{~h}$ and were reported on the computer three times on weekdays, once on Saturday, and not on Sunday.

The figure shows the results for all 736 urine specimens examined. Ninety three per cent of culture negative urine samples and $28 \%$ of culture positive samples were reported in less than 24 hours, and $73 \%$ of culture positives within 48 hours. No reporting on Sunday accounted for most of the remainder. During the week $11(1.5 \%)$ specimens took more than 96 hours to be reported. Inspection of the computer and worksheets of these "outliers" identified the following reasons for delay: (i) failure to notice the specimen remained on an "outstanding worklist" $(n=5)$; (ii) excessive work on non-significant, or mixtures of, organisms $(n=4)$; (iii) justifiable extra technical work on dysgonic or unusually resistant organisms $(\mathrm{n}=2)$.

Only four specimens should reasonably have taken more than three days. The exercise prompted us to change operational aspects of the distribution of the outstanding worklist and to reinforce our rule that the need for detailed work on mixed cultures should be assessed by a medical microbiologist.

Although we determine only a proportion

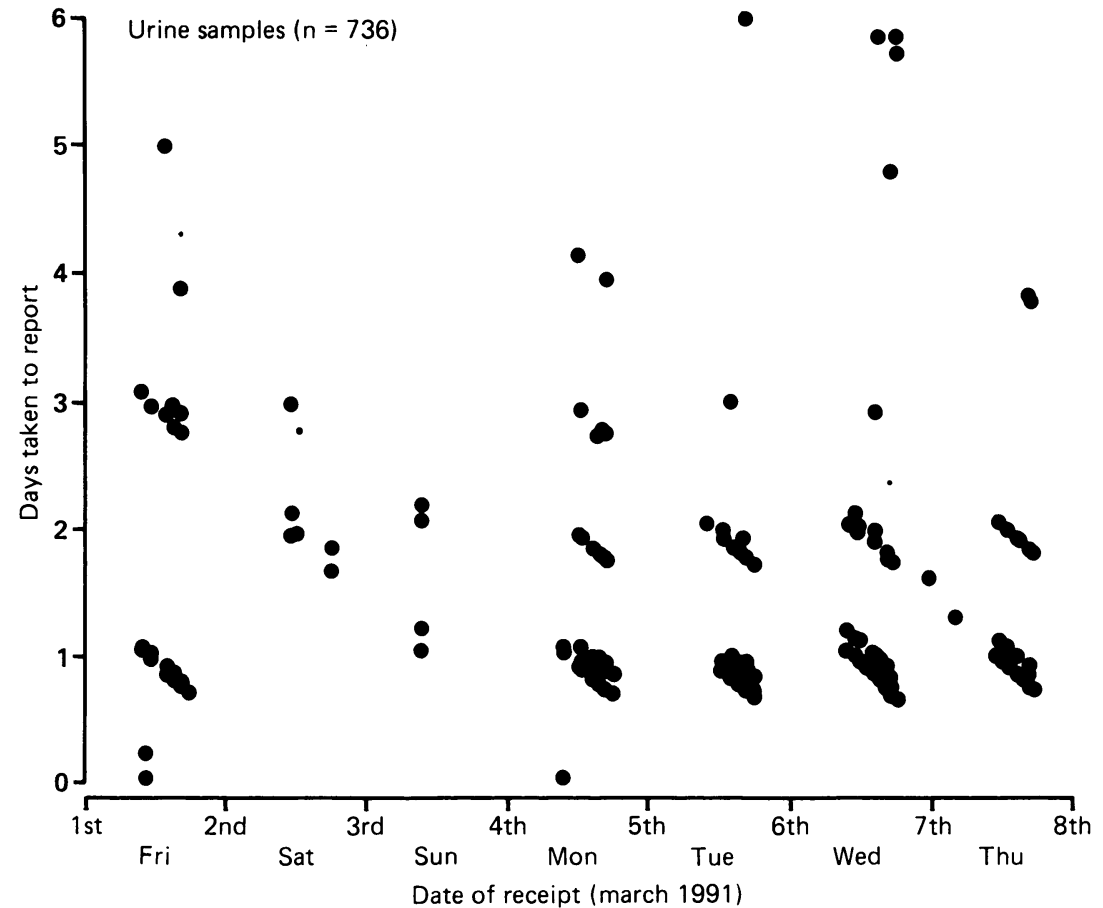

of the full turnround time, efficient use of data captured routinely permits repeated, complete analysis of any group of patients or specimens in an audit process that is flexible, suited to automation, and almost effortless.

WR GRANSDEN

HM MCFARLANE GPJ FELIX Department of Microbiology, St Thomas's Hospital Thomas's Hospital,
London SE1 $7 E H$

1 Rogers S, Bywaters MJ, Reeves DS. Audit of turnround times in a microbiology laboratory. turnround times in a microbiol

\section{Serum alanine transaminase (ALT) reference range in Italy}

We read with interest the article by Goldie and McConnell on ALT reference ranges. ${ }^{1}$

According to a decree of the Italian Ministry of Health last July, only blood products negative for $\mathrm{HBsAg}$, $\mathrm{HIV}$ antibodies, hepatitis $C$ virus antibodies and those showing ALT activities lower than, " 1.5 times the highest value of the normal range of the local population of blood donors," can be transfused. We therefore reassessed the reference range and the cut off point for blood donation of ALT activity (respectively, $<35 \mathrm{IU} / 1$ and $52 \mathrm{IU} / \mathrm{l}$ for both men and women) at the two blood banks served by our laboratory.

We measured ALT in 314 regular blood donors (209 men and 105 women) attending the Legnago blood bank using SMAC II (Technicon) and in 311 regular blood donors (206 men and 105 women) attending the
Nogara blood bank using Ektachem (Kodak). ALT activities, as previously reported, ${ }^{12}$ were skewed to the right, and the results are summarised in the table.

Notwithstanding the differences in the instrumentation and procedure used (pyridoxal phosphate is used only in Ektachem methodology), ALT reference ranges, especially in men, were comparable.

According to the guidelines of the Italian Ministry of Health, we should raise the cut off point for blood donations to $63 \mathrm{IU} / 1$ in Legnago and to $64.5 \mathrm{IU} / 1$ in Nogara for men, and to $42 \mathrm{IU} / 1$ in Legnago and $36 \mathrm{IU} / 1$ in Nogara for women. Our clinical experience and recent reports ${ }^{3}$ suggest that these points are too high. We think that the more cautious 97.5 th percentile cut off point is preferable; all efforts need to be directed towards preventing false negative results, mostly in areas, such as northern Italy, where there are no severe shortages in blood donations.
The geographic, gender, and analytical differences, especially in temperature, of ALT activities preclude extrapolating reference ranges and cut off points for other nations or regions, or the results obtained using alternative instrumentation and methods. ${ }^{34}$ All the blood banks (including those in small or medium sized hospitals) must establish, in cooperation with the local laboratory, a sex specific reference range for ALT. Otherwise, an empirically chosen and unspecific cut off point for blood donations could severely misrepresent the local population of blood donors, obliterating the usefulness of ALT measurements as a "surrogate marker" of non- $A$, non-B hepatitis.

RM DORIZZI F TAGLIARO Clinical Chemistry Laboratory, Hospital of Legnago, Varese, Italy E CAPUZZO Blood bank, Hospital of Legnago, Varese, Italy

Reference values for ALT for Legnago and Nogara blood banks (IU/l)

\begin{tabular}{lll}
\hline & Mean $(S D)$ & $2 \cdot 5-97 \cdot 5$ th percentile \\
\hline Male donors Legnago $(\mathrm{n}=209)$ & $21 \cdot 4(9 \cdot 34)$ & $9-42$ \\
Female donors Legnago $(\mathrm{n}=105)$ & $14 \cdot 7(5 \cdot 72)$ & $7-28$ \\
Male donors Nogara $(\mathrm{n}=206)$ & $20 \cdot 0(9 \cdot 15)$ & $7-43$ \\
Female donors Nogara $(\mathrm{n}=105)$ & $12 \cdot 1(5 \cdot 02)$ & $6-23$ \\
\hline
\end{tabular}

*The results are recorded at $30^{\circ} \mathrm{C}$ using coefficients for $37^{\circ}-30^{\circ} \mathrm{C}$; conversion was experimentally obtained in normal and infected sera. 